$\checkmark$ Research S Suare

\title{
Aberrantly ASF1B Promotes Proliferation and Metastasis of Lung Adenocarcinoma in Vitro Study
}

liyang wu ( $\square$ leonw979@163.com )

Guangzhou Medical University https://orcid.org/0000-0001-6930-1993

\section{Research Article}

Keywords: ASF1B, ASF1A, lung adenocarcinoma, proliferation, metastasis, biomarker

Posted Date: May 25th, 2021

DOI: https://doi.org/10.21203/rs.3.rs-515859/v1

License: (9) This work is licensed under a Creative Commons Attribution 4.0 International License. Read Full License 


\section{Abstract}

ASF1A and ASF1B are two isoforms of the histone $\mathrm{H} 3-\mathrm{H} 4$ chaperone anti-silencing feature 1 (ASF1) found in mammalian cells. To date, however, it remains elusive if they have different physiological functions in lung adenocarcinoma. Here, we identified both ASF1A and ASF1B are elevated in lung adenocarcinoma (LUAD) and lung squamous cell carcinoma (LUSC). ASF1B, but not ASF1A is associated with poor clinical outcomes and act as an independent prognostic factor in LUAD. In vitro studies, knockdown of ASF1B inhibits the proliferation of lung adenocarcinoma through modulating the expression of cyclins and CDK inhibitor (CKI). Moreover, by restraining epithelial-mesenchymal transition (EMT), abnormal of ASF1B could limit metastasis of lung adenocarcinoma. In summary, ASF1B is important in the development of NSCLC and can provide a novel target for lung cancer therapy.

\section{Introduction}

Lung cancer, as a prevalent malignant tumor, caused noticeable mortality and morbidity worldwide[1] . Non-small cell lung cancer (NSCLC), in which lung squamous cell carcinoma (LUSC) and lung adenocarcinoma (LUAD) are two main types, accounts for more than $85 \%$ of all cases[2]. Surgical resection, chemotherapy, and radiation therapy are all common treatments for early-stage NSCLC, but they all have unwanted side effects[3, 4]. Recently, personalized precision medicine, which specifically treats tumor cells based on patients' gene mutations, has gained a lot of therapeutic interest because its less side effects[5]. Patients with NSCLC, on the other hand, have a low prognosis, with a 5-year survival rate of 15.9 percent[4]. As a result, it is important to identify improved prognosis biomarkers for NSCLC.

Histone $\mathrm{H} 3-\mathrm{H} 4$ chaperone anti-silencing feature 1 (ASF1) is a key histone chaperone protein involved in chromatin-based cellular DNA replication, DNA damage repair, and transcription control[6-9]. ASF1A and ASF1B are two paralogs of ASF1 $[6,8]$. ASF1A is primarily involved in DNA repair and cell aging, while ASF1B is primarily involved in cell proliferation[6, 8]. A previous study of Armelle et al. discovered that an elevated ASF1B mRNA level was linked to clinical evidence and disease outcome in breast cancer, and the researchers suggested that ASF1B may be used as a potential proliferation marker for breast cancer detection and prognosis[10]. XJ Liu et al. identified ASF1B promote proliferation and migration in cervical cancer cells, and it could be used as prognostic factor in cervical cancer patients[11]. GY Han et al. clarified ASF1B was highly upregulated in prostate cancer[12]. Moreover, inhibition of ASF1B induced G1 arrest, anti-cell apoptosis, and prevented clonal formation[12]. Recent bioinformatic research found that ASF1B is overexpressed in the TCGA and GEO LUAD databases, and elevated ASF1B expression was correlated with advanced tumor stage and showed prognostic value for LUAD[13].

In the present research, we carried out an analysis for the expression and prognostic value of ASF1A, ASF1B, which identified ASF1B could suitable as an independent prognostic biomarker for LUAD. And ASF1B was expected to LUAD proliferation and lymph node metastasis of LUAD.

\section{Materials And Methods}




\section{TCGA-GTEx Lung Cancer Cohort}

We downloaded The Cancer Genome Atlas (TCGA) - The Genotype-Tissue Expression (GTEx) cohort data from the website of University of California Santa Cruz Xena Browser (https://xenabrowser.net)[14, 15]. Data collation using Excel, we abandoned the recurrent tumor, primary lung adenocarcinoma, primary lung squamous cell carcinoma and solid tissue normal remained for further analysis.

\section{Gene enrichment analysis}

ASF1B co-expression genes were extracted from cBioPortal (http://www.cbioportal.org/) with TCGA LUAD cohort, which has $|r| \geq 0.35$. These co-expressed genes were further carried out Gene Enrichment analysis based upon Kyoto Encyclopedia of Genes (KEGG) in DAVID (https://david.ncifcrf.gov/summary.jsp). Gene sets, which $p<0.05$ were identified as enriched. GSEA was applied to further explored the pathways that correlate to ASF1B expression. Gene sets meets $P<0.05$ and FDR $<0.25$ were considered remarkable enriched.

\section{Cell lines}

The non-neoplastic lung epithelial cell 16HBE, BEAS-A2 and six NSCLC cell lines (A549, H460, H1299, H520, H1975 and SPC-A1) were obtained from the ATCC (American type culture collection). Cell lines were cultured in RPMI-1640 medium (Thermo Fisher Scientific, USA) containing 10\% fetal bovine serum (Gibco, USA). The culture dishes were placed in the incubator at $37^{\circ} \mathrm{C}, 5 \% \mathrm{CO}_{2}$.

\section{Cell infection and transfection}

The siRNA targeting ASF1B and control vector were provided by RiboBio (Guangzhou, China) for si-RNAmediated gene knockdown. The siRNA transfection was performed ASF1B using Lipofectamine 3000 (Thermo Fisher Scientific, Inc.) according to the manufacturer's instructions.

\section{qRT-PCR}

Total RNA was extracted using Trizol RNA reagent (Themo Fisher Scientific, USA). Reverse transcription was performed using the PrimeScript ${ }^{\text {TM }}$ RT reagent Kit with gDNA Eraser (Takara, Japan). qRT-PCR was conducted using SYBR Green ${ }^{\text {TM }}$ Premix Ex Taq ${ }^{\text {TM }}$ II (Takara, Japan) in Applied Biosystems 7500 (AB, USA).

\section{Western blot analysis}

Western blotting was performed according to the standard protocol with the following antibodies:a-tublin, $\beta$-actin, Slug, CDK2/4, CCND1, P27 (CST), Snail, Slug, E-cadherin, Vimentin (Abcam).

\section{Cell counting kit-8 (CCK-8) and colony formation assays}

Cells were planted into 96-well plates with 1000 cells per well. CCK-8 reagents (Djingo, Japan) were added at every at $24 \mathrm{~h}$ for five days. The optical density was estimated at $450 \mathrm{~nm}$ wavelength. Cells were planted 
in in 6-well plates (500/well) and incubation for 2 weeks at $37^{\circ} \mathrm{C}, 5 \% \mathrm{CO}_{2}$. Colonies were washed triple with PBS and stained with crystal violet for $15 \mathrm{~min}$.

\section{Wound Healing assays}

$1 \times 10^{6}$ non-small cell lung cancer cells were routinely seeded and inoculated in a 6-well plate. When the density reached $100 \%$, scratching was conducted with 200 ul sterile tips perpendicularly to the cell plane. Taking photos under the microscope at the time pace: $0 \mathrm{~h}, 24 \mathrm{~h}$.

\section{Migration assays}

Migration assays were performed using transwell chambers (Corning USA). For migration assays, cells $\left(2 \times 10^{5}\right.$ cells $)$ were seeded with serum-free medium onto the top chamber, and the bottom chamber was filled with $10 \%$ FBS medium. After $18 \mathrm{~h}$, all the chambers collected and fixated with methanol for 30 min, stained with $0.01 \%$ crystal violet for $15 \mathrm{~min}$.

\section{Statistically analysis}

For data processing, GraphPad Prism version 8.0 was used. Values in this analysis are described in the form of mean SD (Standard Deviation) unless otherwise specified. Independent survey contrasts between two groups When the two groups' SDs were equal, the Student's t-test (two-tailed) was used, and when the SDs were different, the Student's t-test with Welch's correlation was used. If the variances were equivalent in multigroup samples statistics, one-way ANOVA was used; if not, Welch's ANOVA was used. Both ANOVAs were subjected to Bonferroni post hoc examinations. The TCGA LUAD Cohort samples were split into two categories based on median values. A statistically significant difference was described as a difference of $* P<0.05$, $* \star$ denotes $P<0.01$, *** denotes $P<0.0001$.

\section{Results}

\section{ASF1A and ASF1B are significantly elevated in non-small cell lung cancer tissues compared with normal lung tissues (NTL).}

Expression levels of ASF1 members (ASF1A and ASF1B) were individually analyzed in the TCGA-GTEx lung cancer cohort. In the lung cancer cohort, we observed a distinctly upregulated expression of ASF1A and ASF1B in both LUAD and LUSC samples as compared to that in normal lung tissue (Figure. 1).

Next, we investigated the prognostic importance of elevated ASF1A and ASF1B expression in NSCLC using the TCGA prognostic data (Figure.2A-D). Kaplan-Meier analysis demonstrated that ASF1B mRNA expression was positively correlated with OS, DSS, PFI both in LUAD and LUSC, but only with DFI in LUAD, not in LUSC (Figure.2C-D). However, ASF1A showed no prognostic value in non-small cell lung cancer (Figure.2A-B). 
Moreover, we investigated with the Cox regression, both univariate and multivariate (Figure. 3A-D) process to validate whether ASF1B could serve as an independent prognostic biomarker in lung adenocarcinoma. The results of (Figure.3A-B) present that ASF1B can be a potential predictor for lung adenocarcinoma OS and DSS, rather than DFI and PFI (Figure.3C-D). Considering the meaningful prognostic value of ASF1B, we focused on the function of ASF1B in LUAD for further research.

\section{Correlation of ASF1B expression with the clinicopathological features of NSCLC}

We investigated the association of clinicopathological features of NSCLC with ASF1B mRNA expression to better understand the function of ASF1B expression in NSCLC progression (Figure.4A-F). ASF1B mRNA were related to tumor diameter and lymph node metastasis in the TCGA LUAD cohort, according to our results. Furthermore, there was no substantial correlation between ASF1B mRNA expression with age, sex, distant metastasis, or stage. As a result, we propose that ASF1B promotes LUAD proliferation and lymph node metastasis.

\section{Recognition of the biological processes and pathways associated with ASF1B expression}

To understand the biological functions and potential signaling pathways associated with ASF1B and involved in the tumorigenesis of NSCLC, we performed GSEA using TCGA LUAD cohorts. First, we used the cBioPortal to determine the ASF1B co-expression genes. DAVID was used to analyze the KEGG pathways for genes with $|r| \geq 0.35$. ASF1B modulates cell cycle, DNA replication and p53 signaling pathways, according to the KEGG pathway analysis (Figure.4G-H). GSEA was also used to confirm the mechanisms associated with ASF1B expression. ASF1B was found to be active in the cell cycle, MAPK, and p53 signaling pathways, among others, according to our findings (Figure 3B). On comparing the findings of two enrichment studies, we discovered that they all indicated the involvement of ASF1B in cell cycle and p53 signaling pathways regulation.

\section{Decreased ASF1B inhibits proliferation of lung adenocarcinoma cells}

We investigated ASF1B expression in six different lung cancer cells and human bronchial epithelial cell line with Western blotting. The results indicated ASF1B was highly expressed in six lung cancer lines in protein level, in which A549, PC-9 were the two most high expressed adenocarcinoma cell lines (Figure.S1A). qPCR and western blotting results demonstrated that ASF1B siRNA evidently decreased the expression of ASF1B (Figure.S1B-D). Colony formation and CCK8 proved that knockdown ASF1B significantly blocked proliferation of A549 and PC-9 cells (Figure.5A-B). The western blot results indicated knockdown ASF1B inhibited cyclin-dependent kinases (CDKs) CDK2/4 and Cyclin proteins CCND1, while increased expression of CDK inhibitor (CKI) P27 (Figure.5ㅁ).

\section{ASF1B promotes cell migration of lung adenocarcinoma}

Transwell migration assays and Wound Healing assays demonstrated that knockdown ASF1B inhibited the ability of tumor cell migration in LUAD. (Figure.6A-C). In terms of the mechanism, ASF1B knockdown 
showed features of epithelial-mesenchymal transition (EMT), that decreased N-cadherin, Vimentin, Snail, Slug and increased E-cadherin (Figure.6D).

\section{Discussion}

ASF1 is a member of the histone H3-H4 chaperone protein family that was first discovered in yeast[16]. ASF1A and ASF1B is the two members of ASF1, which controls chromatin functions and has been linked to tumorigenesis[7, 9, 10,17]. However, only several reports have stated the role of ASF1B in tumor, about prostate cancer, breast cancer, and cervical cancer[10-13]. Therein, we have identified ASF1B function as an oncogene in NSCLC through the combination of bioinformatic analysis, molecular biology and cell biological experiments.

ASF1B has been shown to be elevated in many experiments and to be a potential prognostic biomarker. Up-regulated ASF1B expression has been related to a higher risk of disease growth, cancer progression, and metastasis in small breast cancer[10]. Silencing ASF1B in prostate cancer blocked replication, cell cycle arrest, and induced apoptosis, while knocking down ASF1B in cervical cancer was linked to proliferation, migration, and anti-apoptosis[11]. ASF1A and ASF1B were found to be strongly expressed in both LUAD and LUSC samples in the current research. Furthermore, in TCGA samples, overexpressed ASF1B mRNA was negatively associated with OS, DSS, PFI, and DFI in LUAD, but not in LUSC. Furthermore, univariate and multivariate regression analysis confirmed ASF1B mRNA acted as an independent prognostic factor for OS and DSS, but not DFI and PFI in LUAD. Here, we discovered that ASF1B mRNA expression was related to tumor diameters and lymph node metastasis, but not to age, gender, smoking history, metastasis, or clinical stage, after studying the relationship between ASF1B and clinical pathological parameters of lung adenocarcinoma patients. ASF1B could also control proliferation and P53 signaling pathways, according to GSEA and KEGG pathway enrichment studies. ASF1B was shown to encourage proliferation in small breast cancer, prostate cancer, and cervical cancer in previous studies[10-13]. As a result, we hypothesized that ASF1B may play a role in lung adenocarcinoma proliferation and metastasis. Therefore, our hypothesis was that ASF1B may involve in the proliferation and metastasis of lung adenocarcinoma. Reduced ASF1B expression disrupted the proliferation and migration of lung adenocarcinoma cells in vitro trial. ASF1B can restrain lung adenocarcinoma proliferation by controlling the cell cycle, according to the gene enrichment research. According to Western Blot results, ASF1B modulates the cell cycle associated protein.

ASF1B was shown to be linked to cervical cancer migration[11]. Therefore, we hypothesized that ASF1B was involved in lung adenocarcinoma migration. Our Transwell and Wound Healing tests indicated that knockdown ASF1B prevents lung adenocarcinoma cells from migrating. Furthermore, our Western Blot findings revealed that ASF1B regulates migration by reversing EMT.

Our research first establishes the function of ASF1B in the development of NSCLC disease. These findings indicate that ASF1B may be used as a drug target and a prognostic factor in lung 
adenocarcinoma. Consequently, developing highly selective and active ASF1B inhibitors may be crucial in combating chemotherapy resistance and improving lung cancer treatment.

\section{Declarations}

\section{Author Contribution Statement}

L. W. designed the study and experiments, analyzed the data and revised the manuscript, wrote the manuscript, performed all experiments. The author read and approved the final manuscript.

\section{Funding Statement}

Not applicable.

\section{Conflict of Interest Statement}

The authors have no conflicts of interest to declare.

\section{Data Availability Statement}

The datasets used during the present study are available from the corresponding author upon reasonable request. Data were obtained from The Cancer Genome Atlas (TCGA; http://portal.gdc.cancer.gov), GEO database (https://www.ncbi.nlm.nih.gov/geo/) CCLE(https://portals.broadinstitute.org/ccle/about);cBioportal(http://www.cbioportal.org/) and the University of California Santa Cruz Xena Browser (https://xenabrowser.net).

\section{References}

1. Siegel RL, Miller KD, Fuchs HE, Jemal A. Cancer Statistics, 2021. CA: a cancer journal for clinicians. 2021 2021-01-01;71(1):7-33.

2. cancer-facts-and-figures-2021.

3. Molina JR, Yang P, Cassivi SD, Schild SE, Adjei AA. Non-small cell lung cancer: epidemiology, risk factors, treatment, and survivorship. MAYO CLIN PROC. 2008 2008-05-01;83(5):584-94.

4. Hirsch FR, Scagliotti GV, Mulshine JL, Kwon R, Curran WJ, Wu Y, et al. Lung cancer: current therapies and new targeted treatments. The Lancet. 2017;389(10066):299-311.

5. Skoulidis F, Heymach JV. Co-occurring genomic alterations in non-small-cell lung cancer biology and therapy. NAT REV CANCER. 2019;19(9):495-509.

6. Paul PK, Rabaglia ME, Wang CY, Stapleton DS, Leng N, Kendziorski C, et al. Histone chaperone ASF1B promotes human beta-cell proliferation via recruitment of histone H3.3. CELL CYCLE. 2016 2016-12-01;15(23):3191-202.

7. Avvakumov N, Nourani A, Côté J. Histone Chaperones: Modulators of Chromatin Marks. MOL CELL. 2011 2011-01-01;41(5):502-14. 
8. Messiaen S, Guiard J, Aigueperse C, Fliniaux I, Tourpin S, Barroca V, et al. Loss of the histone chaperone ASF1B reduces female reproductive capacity in mice. REPRODUCTION. 2016 2016-0501;151(5):477-89.

9. Peng H, Nogueira ML, Vogel JL, Kristie TM. Transcriptional coactivator HCF-1 couples the histone chaperone Asf1b to HSV-1 DNA replication components. Proc Natl Acad Sci U S A. 2010 2010-0209;107(6):2461-6.

10. Corpet A, De Koning L, Toedling J, Savignoni A, Berger F, Lemaitre C, et al. Asf1b, the necessary Asf1 isoform for proliferation, is predictive of outcome in breast cancer. EMBO J. 2011 2011-0202;30(3):480-93.

11. Liu X, Song J, Zhang Y, Wang H, Sun H, Feng X, et al. ASF1B promotes cervical cancer progression through stabilization of CDK9. CELL DEATH DIS. 2020;11(8).

12. Han G, Zhang X, Liu P, Yu Q, Li Z, Yu Q, et al. Knockdown of anti-silencing function 1B histone chaperone induces cell apoptosis via repressing PI3K/Akt pathway in prostate cancer. INT J ONCOL. 2018 2018-01-01;53(5):2056-66.

13. Feng Z, Zhang J, Zheng Y, Wang Q, Min X, Tian T. Elevated expression of ASF1B correlates with poor prognosis in human lung adenocarcinoma. PERS MED. 2021 2021-01-01;18(2):115-27.

14. Keen J, Moore H. The Genotype-Tissue Expression (GTEx) Project: Linking Clinical Data with Molecular Analysis to Advance Personalized Medicine. Journal of Personalized Medicine. 2015 2015-02-05;5(1):22-9.

15. Lonsdale J, Thomas J, Salvatore M, Phillips R, Lo E, Shad S, et al. The Genotype-Tissue Expression (GTEx) project. NAT GENET. 2013;45(6):580-5.

16. Kobayashi R, Kamakaka RT, Adams CR, Kadonaga JT, Chen S, Tyler JK. The RCAF complex mediates chromatin assembly during DNA replication and repair. Nature (London). 1999 1999-0101;402(6761):555-60.

17. 17. O'Sullivan RJ, Arnoult N, Lackner DH, Oganesian L, Haggblom C, Corpet A, et al. Rapid induction of alternative lengthening of telomeres by depletion of the histone chaperone ASF1. NAT STRUCT MOL BIOL. 2014;21(2):167-74.

\section{Figures}


A GTEx-TCGA-LUAD

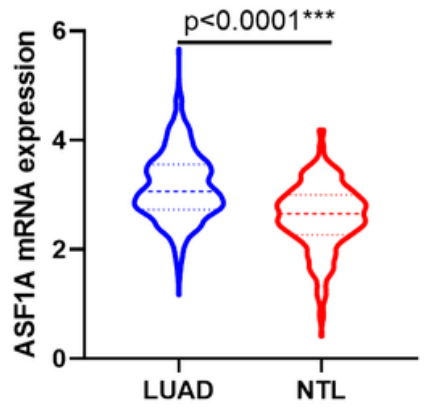

B GTEX-TCGA-LUAD

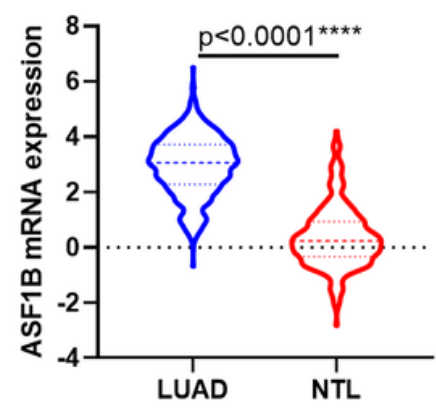

C GTEX-TCGA-LUSC

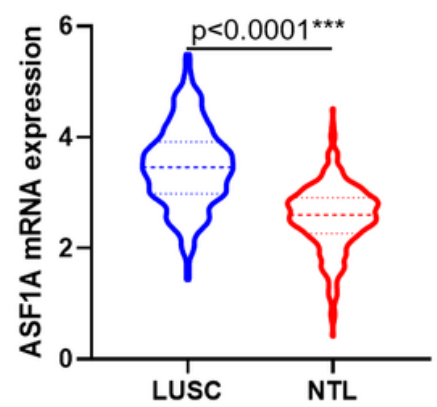

D GTEX-TCGA-LUSC

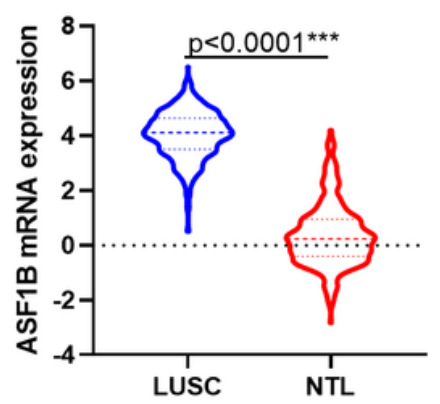

Figure 1

ASF1A and ASF1B is upregulated in lung cancer (A-D). ASF1A and ASF1B mRNA expression in lung adenocarcinoma and lung squamous cell carcinoma compared with normal lung tissues based on samples from GTEx-TCGA database. 
A

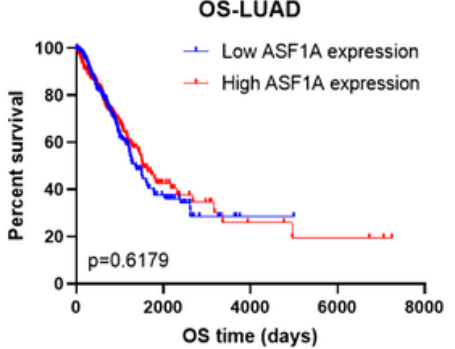

B

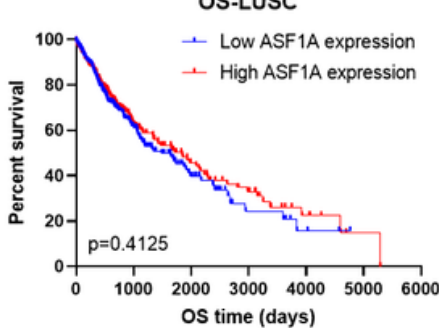

$\mathrm{C}$

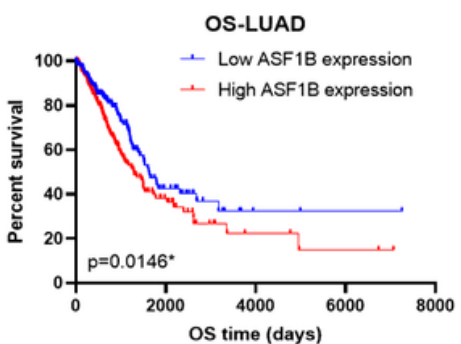

D

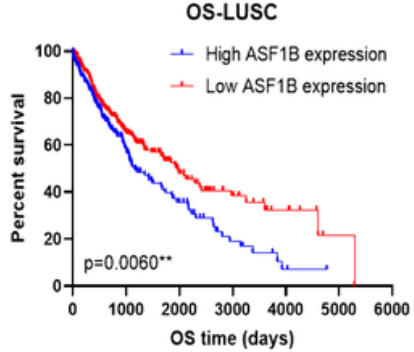

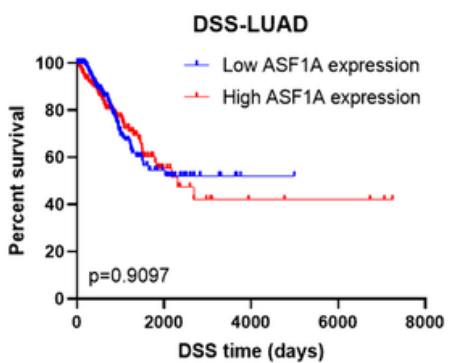
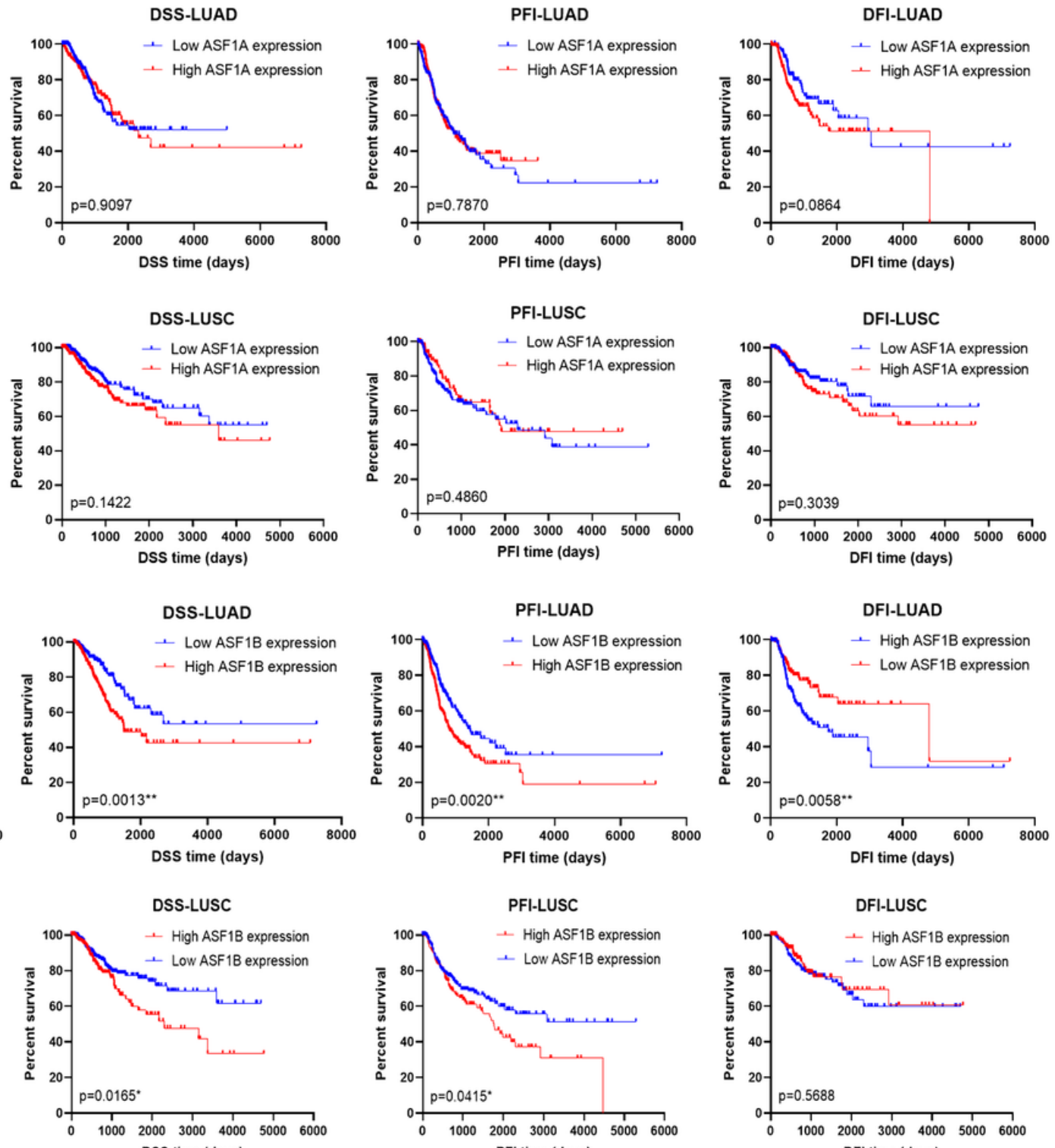

DSS time (days)
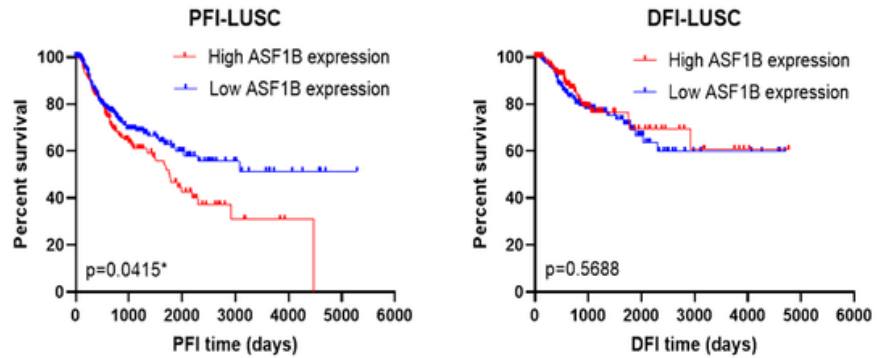

Figure 2

Overexpressed ASF1B confers poor prognosis for lung adenocarcinoma. (A-D). Kaplan-Meier OS, DSS, DFI and PFI survival analysis of lung adenocarcinoma and lung squamous cell carcinoma patients from TCGA database based on ASF1A and ASF1B expression. 

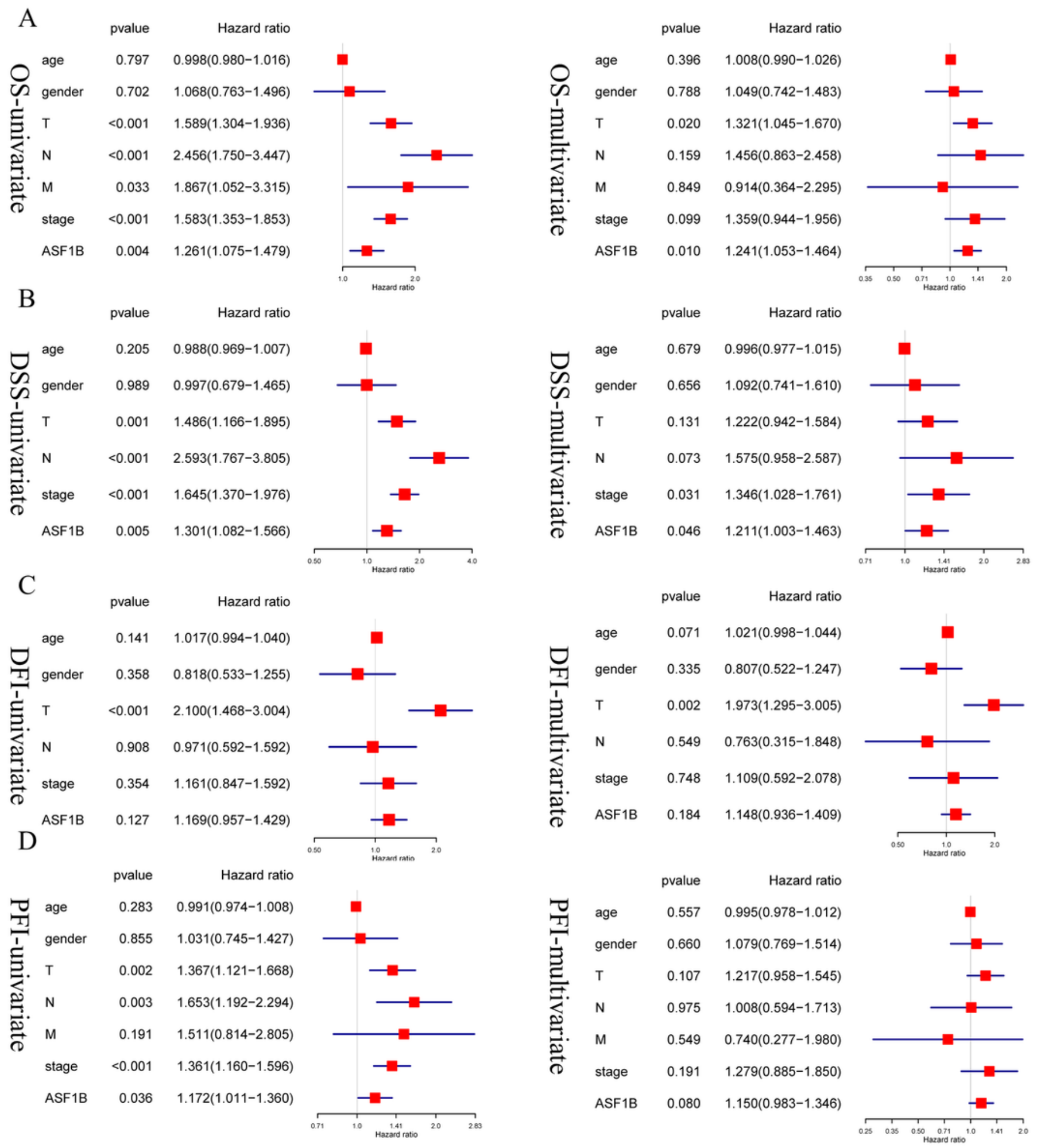

\section{Figure 3}

Overexpressed ASF1B is an independent prognostic biomarker of OS, DSS for patients with lung adenocarcinomas patients. (A-D). The Univariate and Multivariate analysis of OS, DSS, DFI, PFI and clinical-pathological features in TCGA lung adenocarcinoma patients. 
A

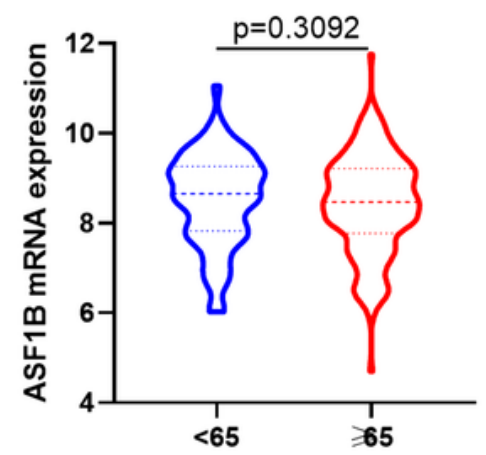

D

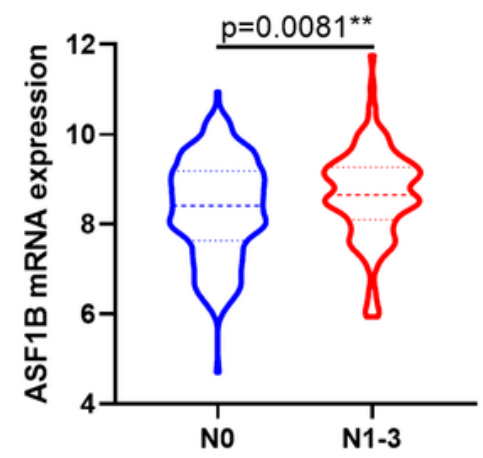

G

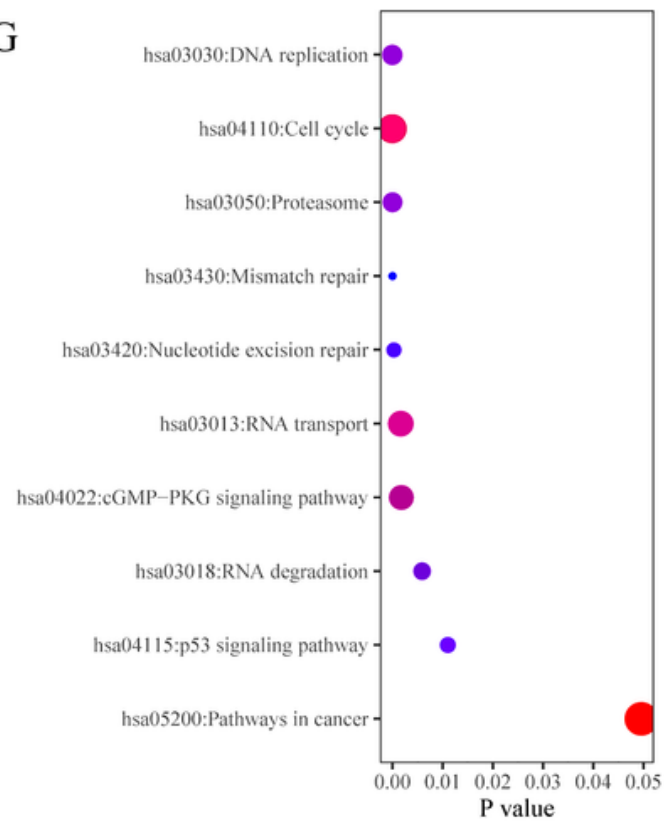

B Gender

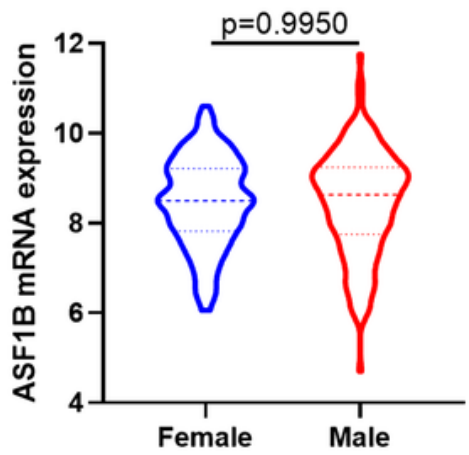

E

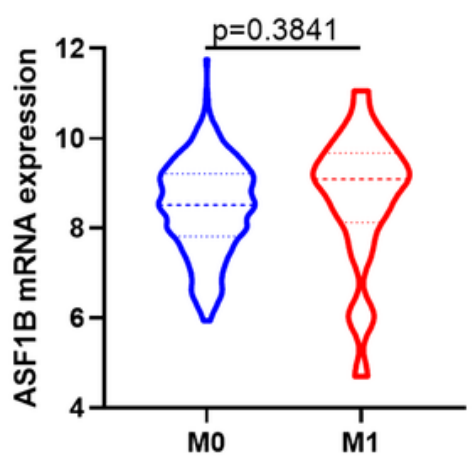

C T

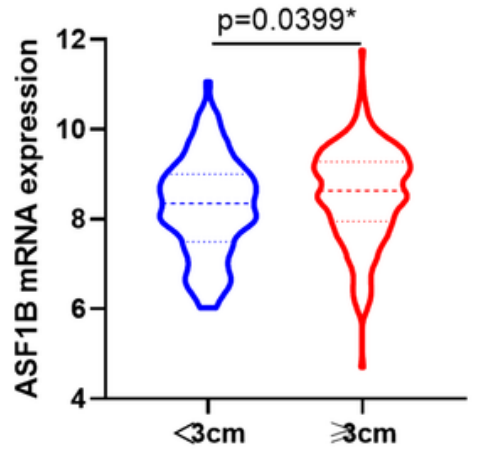

F Stage

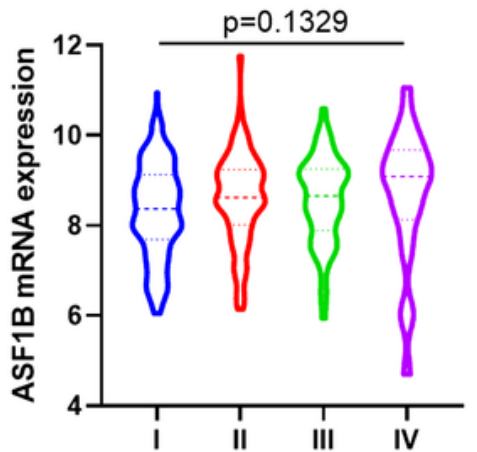

\section{Figure 4}

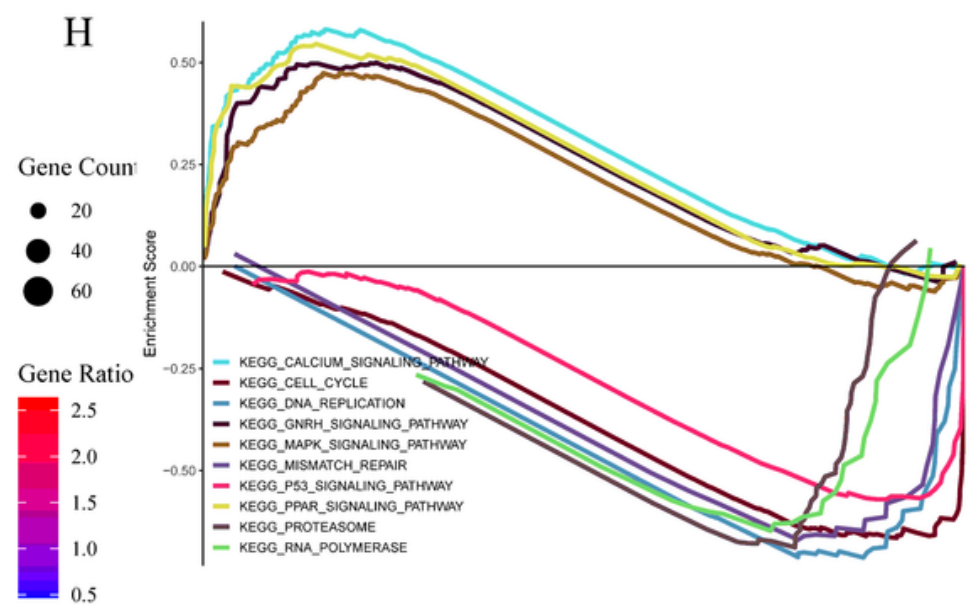

The function of ASF1B in lung adenocarcinoma. (A-F) The correlation of ASF1B mRNA expression and clinical parameters of lung adenocarcinomas. Gene Enrichment Analysis based KEGG pathways $(G)$ and Gene Ontology $(\mathrm{H})$ utilized co-expression genes. 


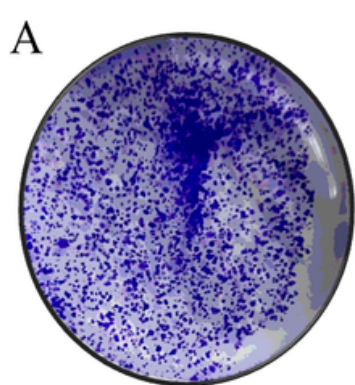

A549-NC

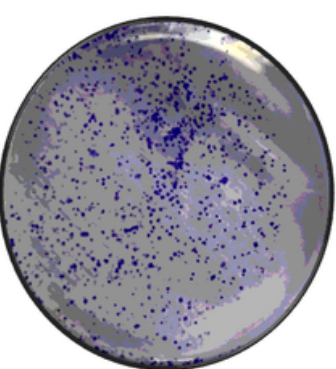

A549-siASF1B

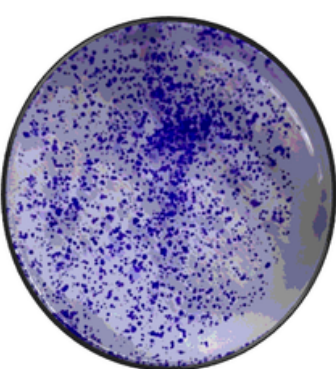

PC-9-NC

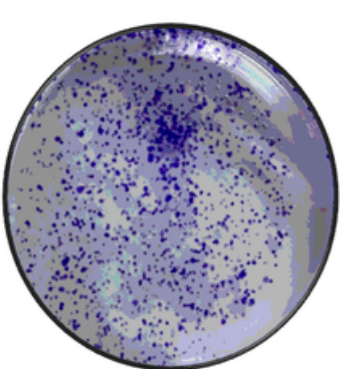

PC-9-siASF1B
B

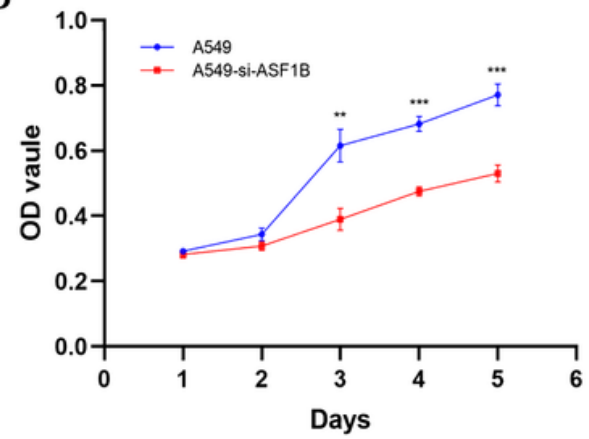

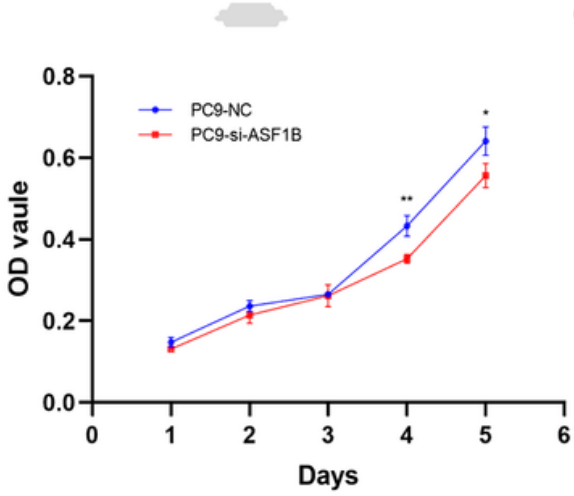

$\mathrm{C}$

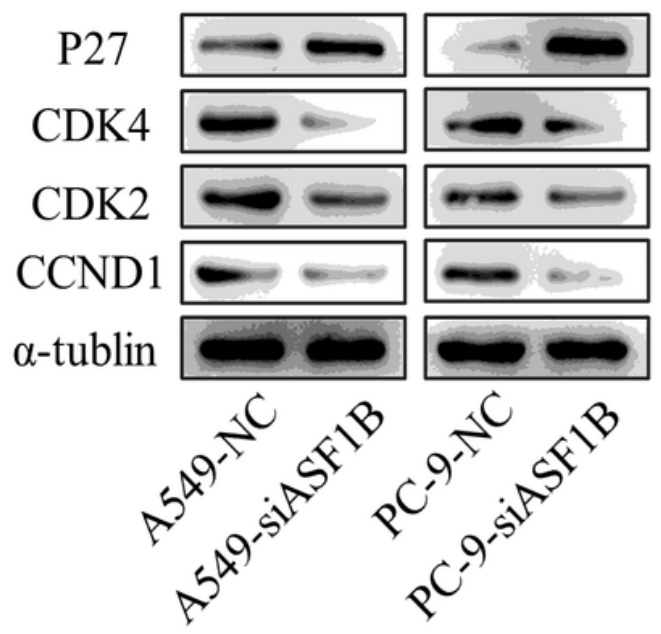

Figure 5

ASF1B promote proliferation of lung adenocarcinomas. (A, D) Colony formation assays, (B) CCK-8 assays prove knockdown ASF1B inhibit proliferation of lung adenocarcinomas. (C). Western blot analysis was used to examine proliferation signaling-associated protein expression levels in lung adenocarcinoma cells. 
A
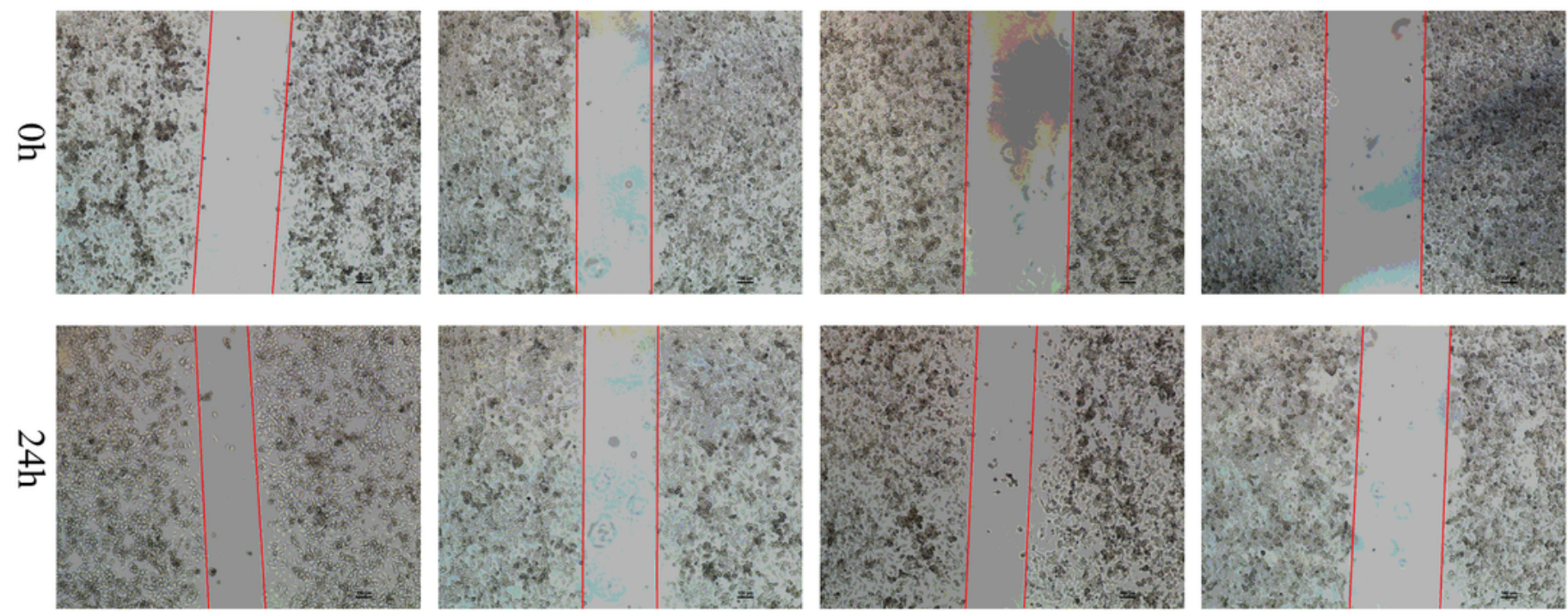

A549-NC

A549-siASF1B

PC-9-NC

PC-9-siASF1B

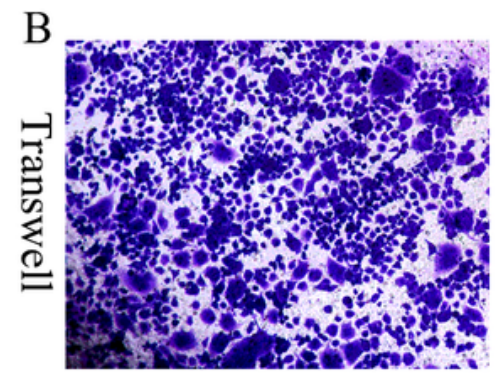

A549-NC

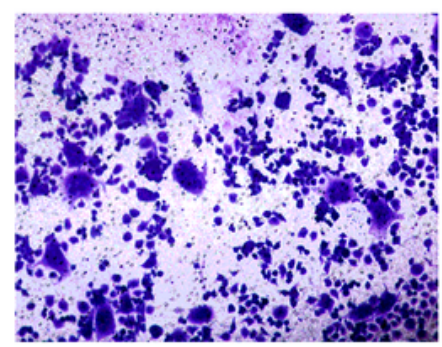

A549-siASF1B

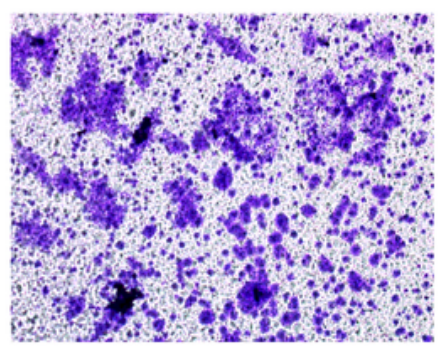

PC-9-NC

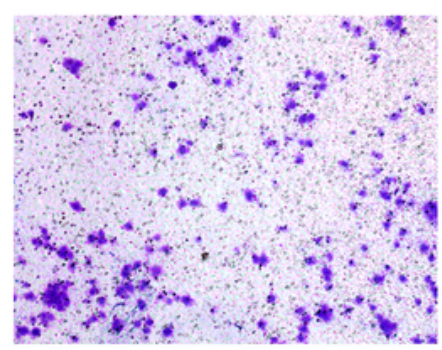

PC-9-siASF1B
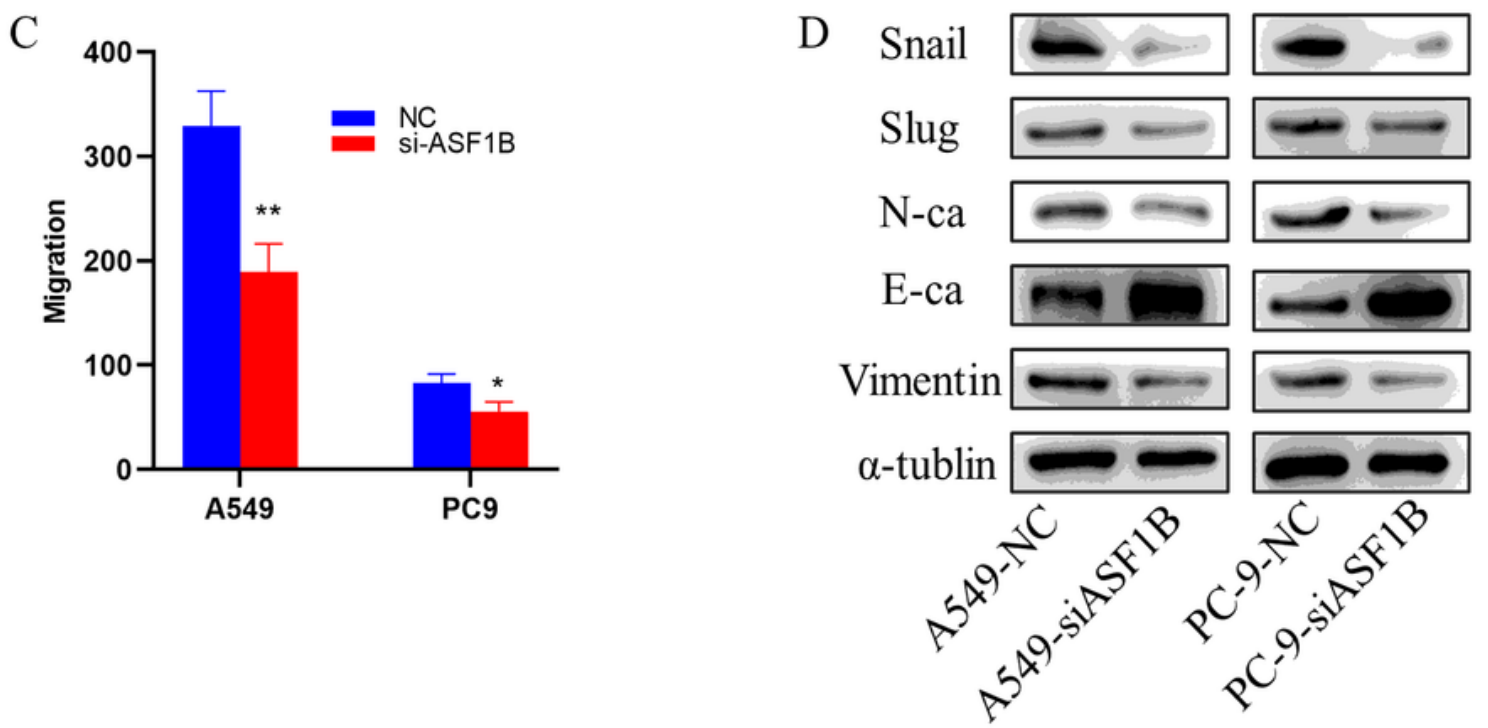

\section{Figure 6}

ASF1B promote migration of lung adenocarcinomas. (A) Wound Healing, (B-C) Transwell assays demonstrated ASF1B-konckdown block migration of lung adenocarcinomas cells. (D). Western blot analysis was utilized to examine metastasis signaling-associated protein expression levels in lung adenocarcinoma cells. 